\title{
Recent cross-section, diffractive and forward multiplicity measurements with TOTEM
}

\author{
Kenneth Österberg* on behalf of the TOTEM collaboration \\ Department of Physics, University of Helsinki and Helsinki Institute of Physics, Finland \\ E-mail: kenneth.osterbergehelsinki.fi
}

\begin{abstract}
TOTEM is dedicated experiment at the LHC to the measurements of total cross-section, elastic scattering and diffraction. Elastically and diffractively scattered protons are measured in Roman Pots and charged particles by the $\mathrm{T} 1$ and $\mathrm{T} 2$ telescopes in the pseudorapidity range 3.1 to 6.5 . TOTEM has measured the luminosity-independent total, elastic and inelastic proton-proton crosssections at $\sqrt{s}=7 \mathrm{TeV}$ and $8 \mathrm{TeV}$ using low-pileup runs with a dedicated $\beta^{*}=90 \mathrm{~m}$ optics. At $\sqrt{s}$ $=7 \mathrm{TeV}$, the cross-sections were also determined using the CMS luminosity giving results that are in excellent agreement with the luminosity-independent ones, despite having very different systematic dependencies. Moreover, since TOTEM is capable to detect diffractive events with masses down to $3.4 \mathrm{GeV}$, a limit on the cross section for low mass diffraction has been set from the difference between the overall inelastic cross section (measured only using elastic scattering) and visible inelastic cross section (measured using $\mathrm{T} 1$ and $\mathrm{T} 2$ ). The differential elastic protonproton cross section has been measured over a wide range in $|t|$ for $\sqrt{s}=7 \mathrm{TeV}$. TOTEM has also determined double diffractive cross-section in the forward region at $\sqrt{s}=7 \mathrm{TeV}$ as well as studied soft single diffraction. In addition, TOTEM has performed an inclusive forward charged particle pseudorapdity density measurement in $7 \mathrm{TeV}$ proton-proton collisions for the range $5.3<|\eta|<$ 6.4 using T2. Next, TOTEM will together with CMS measure the charged particle pseudorapidity density at $\sqrt{s}=8 \mathrm{TeV}$ simultaneously in the central (CMS tracker) and forward (T2) region.
\end{abstract}

The European Physical Society Conference on High Energy Physics -EPS-HEP2013

18-24 July 2013

Stockholm, Sweden

\footnotetext{
* Speaker.
} 


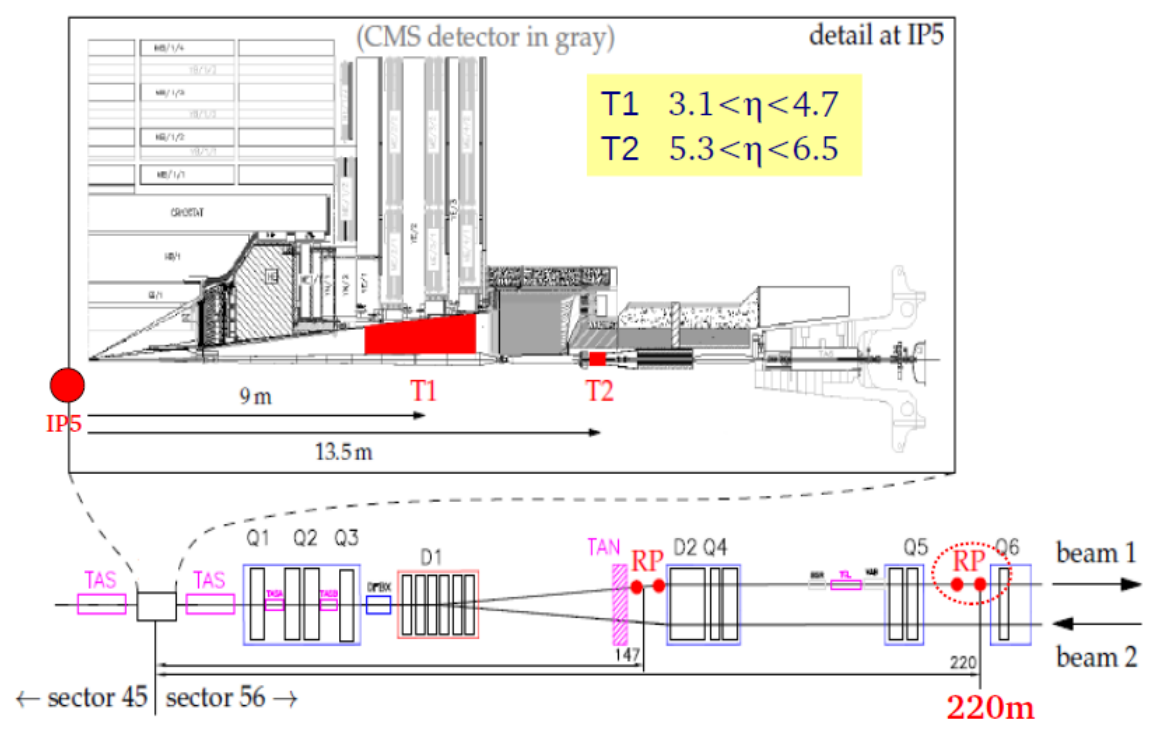

Figure 1: Top: the T1 and T2 telescopes embedded in the forward region of CMS. Bottom: the location of the Roman Pots along the LHC beam line. All detectors are installed symmetrically with respect to IP5.

\section{The TOTEM experiment}

The TOTEM experiment [四] is dedicated to forward hadronic phenomena at the LHC. The three pillars of its physics programme are: an accurate determination of the total cross-section $\left(\sigma_{\mathrm{tot}}\right)$, a measurement of differential elastic scattering cross-section $\left(\mathrm{d} \sigma_{\mathrm{el}} / \mathrm{d} t\right)$ in a wide $t$-range and studies of diffractive processes, partially in cooperation with the CMS experiment [ []].

The physics programme puts special requirements on the detector apparatus. In particular, large pseudorapidity $\eta$ coverage (to detect most inelastic collisions) and excellent acceptance for diffractive and elastic protons. To accomplish this task, TOTEM comprises three subdetectors: the inelastic telescopes T1 and T2 and Roman Pots (RP) for leading proton detection (see Fig. 四).

$\mathrm{T} 1$ and T2 are embedded in the forward region of CMS (Fig. $\mathbb{W}$ ) on each side of the LHC interaction point (IP) 5. The T1 consists of Cathode Strip Chambers (CSC) and T2 of Gas Electron Multipliers (GEM). The $\eta$-coverages of $\mathrm{T} 1$ and $\mathrm{T} 2$ are $3.1 \leq|\eta| \leq 4.7$ and $5.3 \leq|\eta| \leq 6.5$, respectively. $\mathrm{T} 1$ and $\mathrm{T} 2$ are good for defining rapidity gaps, i.e. $\eta$-ranges without primary particles, due to their low transverse momentum $p_{\mathrm{T}}$ threshold, $\sim 100 \mathrm{MeV} / \mathrm{c}$ and $\sim 40 \mathrm{MeV} / \mathrm{c}$ for T1 and T2, respectively. In addition, the $\mathrm{T} 2$ provides an efficient trigger for inelastic events, since more than $90 \%$ of the inelastic events contain at least one charged particle within the T2 acceptance.

The RP system consists of two stations placed at $+220 \mathrm{~m}$ (right arm) and $-220 \mathrm{~m}$ (left arm) from IP5. Each of the stations is composed of two units (far and near with respect to IP5) separated by $\sim 5 \mathrm{~m}$ that is beneficial for proton kinematics reconstruction and background discrimination. Each unit includes two vertical (top and bottom) and one horizontal RP. The RPs are movable beam-pipe insertions that can bring sensitive detectors to sub-millimetre distance from the beam once it is stable. Each RP hosts 5 back-to-back mounted pairs of silicon strip sensors with reduced $(\sim 50 \mu \mathrm{m})$ insensitive edge facing the beam, not to lose protons scattered at very low angles. 


\section{Total, elastic and inelastic pp cross section}

Elastic scattering events consists of two back-to-back protons in the vertical RPs with a diagonal topology: the left arm proton in the bottom RP and the right arm proton in the top RP, or vice versa. Key ingredients are a good understanding of the RP alignment and the LHC optics. In addition to topology, the elastic events were selected based on the collinearity of the protons and their compatibility of being protons with no momentum loss and coming from the same vertex.

Another ingredient in $\sigma_{\text {tot }}$ measurements is the inelastic rate. The rate is determined by counting the $\mathrm{T} 2$ triggered events and correcting for $\mathrm{T} 2$ trigger and reconstruction inefficiencies, beam-gas background, pileup and events with particles only in T1, almost exclusively determined from the data. To obtain the overall inelastic rate, a correction for events with all final-state particles beyond the instrumented region is done based on phenomenological models for low mass diffraction. In the case of TOTEM, the model dependence is minimal due to the excellent pseudorapidity coverage of the T2, allowing the detection of diffractive masses larger than 3.4 (3.6) GeV for 7 (8) TeV.

TOTEM performed a first measurement of $\mathrm{d} \sigma_{\mathrm{el}} / \mathrm{d} t$ at $\sqrt{s}=7 \mathrm{TeV}$ in the $0.36<|\mathrm{t}|<2.5 \mathrm{GeV}^{2}$ range using data taken in 2010 with the standard LHC optics $\left(\beta^{*}=3.5 \mathrm{~m}\right)$, during a dedicated low pileup run [B]]. In June 2011, with a dedicated beam optics configuration $\left(\beta^{*}=90 \mathrm{~m}\right)$, the elastic scattering rate $\left(N_{\mathrm{el}}\right)$ was measured down to $\left|t_{\min }\right|=2 \cdot 10^{-2} \mathrm{GeV}^{2}$ [四], with $\sim 67 \%$ of the full $N_{\mathrm{el}}$. The total $N_{\mathrm{el}}$ was estimated as the sum of the detected rate and the one integrated between $|\mathrm{t}|=0$ and $\left|t_{\min }\right|$ assuming an exponential, $A e^{-B|t|}$, with $B$ determined on the measured data for $|\mathrm{t}|<0.2$ $\mathrm{GeV}^{2}$. With the use of the optical theorem, the CMS luminosity ( $\mathscr{L}$, known with $4 \%$ uncertainty) and the $\rho$ value $^{1}$ of COMPETE [[]], the elastic, total and inelastic cross section was determined:

$$
\sigma_{\mathrm{tot}}^{2}=\left.\frac{16 \pi(\hbar c)^{2}}{1+\rho^{2}} \frac{1}{\mathscr{L}} \frac{d N_{\mathrm{el}}}{d t}\right|_{t=0}, \quad \sigma_{\mathrm{inel}}=\sigma_{\mathrm{tot}}-\sigma_{\mathrm{el}} .
$$

The analysis was also repeated with a low luminosity run at $\beta^{*}=90 \mathrm{~m}$ taken in October 2011, where even smaller |t| values, down to $5 \cdot 10^{-3} \mathrm{GeV}^{2}$, were measured. This enabled the observation of 91 $\%$ of $N_{\mathrm{el}}$, allowing an improved extrapolation to the optical point [6]. A summary of the TOTEM differential elastic cross-section measurements at $\sqrt{s}=7 \mathrm{TeV}$ is shown in Fig. \.

For $|\mathrm{t}|<0.2 \mathrm{GeV}^{2}$, the data is very well described by an exponential with slope $B=19.9 \pm$ $0.3 \mathrm{GeV}^{-2}$. The diffractive minimum was found at $\left|t_{\text {dip }}\right|=0.53 \pm 0.01 \mathrm{GeV}^{2} . B$ and $\left|t_{\text {dip }}\right|$ are found to be larger and smaller, respectively, with respect to the values obtained at lower $\sqrt{s}$. Therefore TOTEM has been able to confirm the "shrinkage of the diffractive peak" in pp elastic scattering with increasing centre-of-mass energy. The luminosity-dependent total, elastic and inelastic cross section, obtained by measuring the elastic rate $N_{\mathrm{el}}$ only, were found to be [6]:

$$
\sigma_{\text {tot }}=98.6 \pm 2.2 \mathrm{mb} \quad \sigma_{\mathrm{el}}=25.4 \pm 1.1 \mathrm{mb} \quad \sigma_{\text {inel }}=73.2 \pm 1.3 \mathrm{mb}
$$

With the same data as in [可], $\sigma_{\text {inel }}$ was also directly measured using inelastic events triggered by the T2 telescope, with the luminosity provided by CMS [四]. The total inelastic cross-section was determined to be $73.7 \pm 3.4 \mathrm{mb}$. If the correction for low mass diffraction was not applied, a cross-section for inelastic events with at least one $|\eta|<6.5$ final-state particle, $\sigma_{\text {inel },|\eta|<6.5}=70.5$ $\pm 2.9 \mathrm{mb}$, was obtained. Subtracting $\sigma_{\text {inel },|\eta|<6.5}$ from $\sigma_{\text {inel }}$ of Eq. (2.2) based only on elastic scattering, an estimate for low mass diffraction i.e. the cross-section for inelastic events with only $|\eta|$

\footnotetext{
${ }^{1} \rho=\Re A_{\mathrm{el}}^{\text {nucl }}(\mathrm{t}=0) / \mathfrak{S} A_{\mathrm{el}}^{\text {nucl }}(\mathrm{t}=0)$, where $A_{\mathrm{el}}^{\text {nucl }}(\mathrm{t}=0)$ is the nuclear elastic amplitude at $\mathrm{t}=0$.
} 


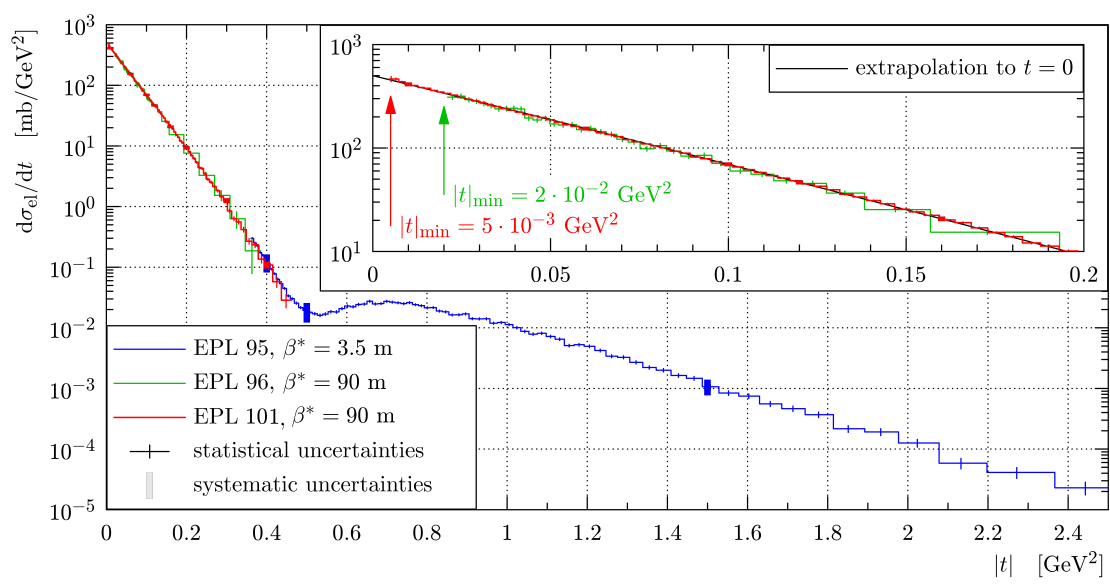

Figure 2: The $|t|$-differential elastic cross section at $\sqrt{s}=7 \mathrm{TeV}$. Inserted: a blowup of the low- $|t|$ region.

$>6.5$ final-state particles, $\sigma_{\text {inel, },|\eta|>6.5}=2.62 \pm 2.17 \mathrm{mb}$ was determined. Note the uncertainty is dominated by the uncertainty on the luminosity. From it, an upper limit of $6.3 \mathrm{mb}$ at $95 \%$ confidence level on the cross-section for events with diffractive masses below $3.4 \mathrm{GeV}$ was deduced. The measurement of the luminosity independent total cross section was obtained from the relation:

$$
\sigma_{\mathrm{tot}}^{2}=\left.\frac{16 \pi(\hbar c)^{2}}{1+\rho^{2}} \frac{1}{N_{\mathrm{el}}+N_{\mathrm{inel}}} \frac{d N_{\mathrm{el}}}{d t}\right|_{t=0} .
$$

where $N_{\mathrm{el}}$ and $N_{\text {inel }}$ are the elastic and inelastic rates integrated over the same data taking period. The total, elastic and inelastic luminosity-independent cross section were found to be [8]:

$$
\sigma_{\text {tot }}=98.0 \pm 2.5 \mathrm{mb} \quad \sigma_{\mathrm{el}}=25.1 \pm 1.1 \mathrm{mb} \quad \sigma_{\text {inel }}=72.9 \pm 1.5 \mathrm{mb}
$$

Determined from a $\beta^{*}=90 \mathrm{~m}$ run in July 2012 with the elastic proton $|t|$ measured down to $\left|t_{\min }\right|=$ $10^{-2} \mathrm{GeV}^{2}$, the luminosity-independent cross sections at $\sqrt{s}=8 \mathrm{TeV}$ were found to be [Q]]:

$$
\sigma_{\mathrm{tot}}=101.7 \pm 2.9 \mathrm{mb} \quad \sigma_{\mathrm{el}}=27.1 \pm 1.4 \mathrm{mb} \quad \sigma_{\text {inel }}=74.7 \pm 1.7 \mathrm{mb}
$$

The cross section measurements at 7 and $8 \mathrm{TeV}$, in good agreement with the COMPETE extrapolation, are summarized in Fig. [3. The right part of Fig. [3] shows a comparison of the cross section measurements at $\sqrt{s}=7 \mathrm{TeV}$ obtained with different methods [8]]: in $(1,2)$ the optical theorem, the luminosity from CMS and $\rho$ from COMPETE were used, for two runs with different instantaneous luminosity; in (3) a $\rho$-independent measurement was performed, using the luminosity from CMS and the relation $\sigma_{\mathrm{tot}}=\sigma_{\mathrm{el}}+\sigma_{\text {inel }}$; in (4) the cross sections were evaluated with the luminosity-independent method. (1) and (2) included no assumption on low mass diffraction, (3) no $\rho$ input and didn't rely on the validity of the optical theorem and (4) no $\mathscr{L}$ input.

Note that an elastic and total cross section analysis at $8 \mathrm{TeV}$ is ongoing based on a low luminosity run with $\beta^{*}=1 \mathrm{~km}$, where elastic protons were measured down to $\left|\mathrm{t}_{\text {min }}\right|=6 \cdot 10^{-4} \mathrm{GeV}^{2}$ allowing a detailed study of the nuclear-Coulomb interference. In addition, analysis of data taken with $\beta^{*}=11 \mathrm{~m}$ at $\sqrt{s}=2.76 \mathrm{TeV}$ in 2013 is on-going. With this optics, elastic protons down to $\left|\mathrm{t}_{\text {min }}\right|$ $=6 \cdot 10^{-2} \mathrm{GeV}^{2}$ were measured, sufficient to allow a luminosity-independent $\sigma_{\text {tot }}$ measurement. 


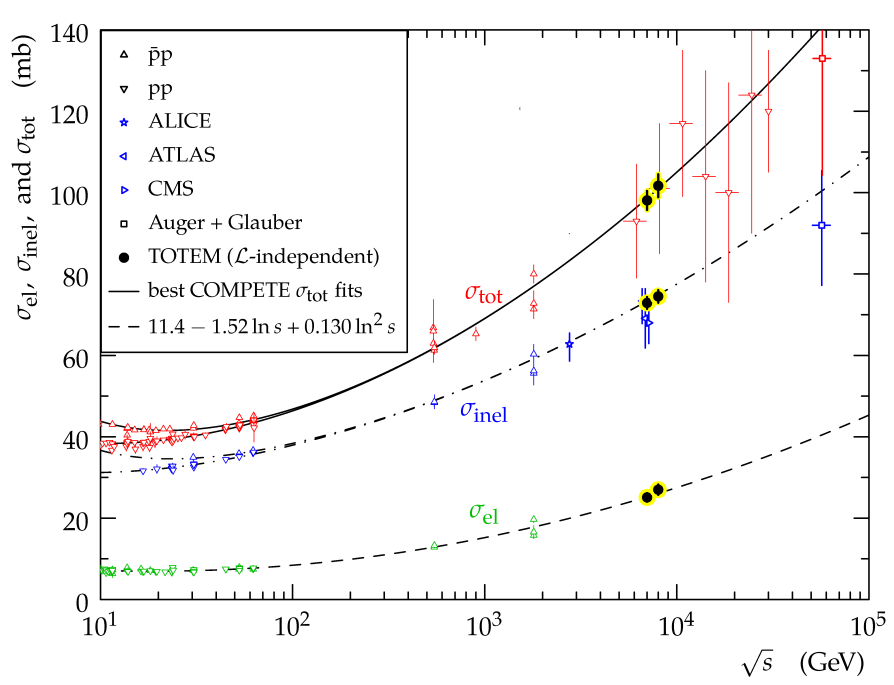

Measurements at $\sqrt{s}=7 \mathrm{TeV}$
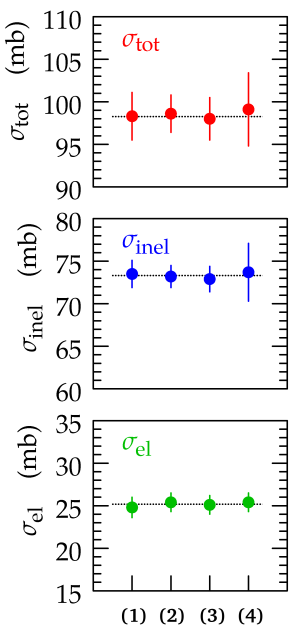

Figure 3: Left: measurement of the luminosity-independent $\sigma_{\mathrm{tot}}, \sigma_{\mathrm{el}}, \sigma_{\text {inel }}$ at $\sqrt{s}=7$ and $8 \mathrm{TeV}$. Measurements from cosmic rays, other LHC experiments and lower energy data are also superimposed together with the best fits from the COMPETE collaboration [ [ $]$ ]. Right: comparison of the TOTEM measurement $\sigma_{\text {tot }}$, $\sigma_{\mathrm{el}}, \sigma_{\text {inel }}$ obtained with different analysis methods (see text for more details).

Table 1: The forward double diffractive (DD) cross-section measurements. $\left|\eta^{+}\right|_{\min }\left(\left|\eta^{-}\right|_{\min }\right)$ refers to the primary particle with smallest $|\eta|$ in the positive (negative) hemisphere. The measurements are compared to MC predictions. The Pythia and Phojet estimates for total $\sigma_{\mathrm{DD}}$ are $8.1 \mathrm{mb}$ and $3.9 \mathrm{mb}$, respectively.

\begin{tabular}{lccc}
\hline & TOTEM & Pythia8.108 & Phojet1.12 \\
\hline \hline $4.7<\left|\eta^{+}\right|_{\min }<6.5$ and $-4.7>-\left|\eta^{-}\right|_{\min }>-6.5$ & $116 \pm 25 \mu \mathrm{b}$ & $159 \mu \mathrm{b}$ & $101 \mu \mathrm{b}$ \\
\hline $4.7<\left|\eta^{+}\right|_{\min }<5.9$ and $-4.7>-\left|\eta^{-}\right|_{\min }>-5.9$ & $65 \pm 20 \mu \mathrm{b}$ & $70 \mu \mathrm{b}$ & $44 \mu \mathrm{b}$ \\
\hline $5.9<\left|\eta^{+}\right|_{\min }<6.5$ and $-5.9>-\left|\eta^{-}\right|_{\min }>-6.5$ & $12 \pm 5 \mu \mathrm{b}$ & $17 \mu \mathrm{b}$ & $12 \mu \mathrm{b}$ \\
\hline $4.7<\left|\eta^{+}\right|_{\min }<5.9$ and $-5.9>-\left|\eta^{-}\right|_{\min }>-6.5$ & $26 \pm 5 \mu \mathrm{b}$ & $36 \mu \mathrm{b}$ & $23 \mu \mathrm{b}$ \\
\hline $5.9<\left|\eta^{+}\right|_{\min }<6.5$ and $-4.7>-\left|\eta^{-}\right|_{\min }>-5.9$ & $27 \pm 5 \mu \mathrm{b}$ & $36 \mu \mathrm{b}$ & $23 \mu \mathrm{b}$
\end{tabular}

\section{Double diffractive cross section and studies of single diffraction}

The double diffractive (DD) cross section in the forward region [ए0] was measured using the same $\beta^{*}=90 \mathrm{~m}$ run at $7 \mathrm{TeV}$ as (2)-(4) in Fig. [3. A forward DD sample was obtained by triggering with $\mathrm{T} 2$, requiring at least one charged particle in both $\mathrm{T} 2$ arms and no charged particles in $\mathrm{T} 1$. This enables a very pure $(\sim 70 \%)$ DD sample to be selected even though it only represents a few $\%$ of the total $\sigma_{\mathrm{DD}}$. The measurement was corrected in order to be representative for DD events with a central rapidity gap between $\eta=-4.7$ and $\eta=+4.7$. This corresponds approximately to $\sigma_{\mathrm{DD}}$ of events, where both diffractive systems have masses between 3.4 and $8 \mathrm{GeV}$.

The non diffractive (ND) background was estimated by scaling the MC predictions according to a control sample from data dominated by ND (with charged particles in both T1 and T2 
Table 2: Classification of the single diffractive events into different ranges of the diffractive mass ("opposite" refers here to the opposite side of the IP with respect to the proton in the RP).

\begin{tabular}{cccc}
\hline SD class & Inelastic telescope configuration & Mass $\left(M_{\text {diff }}\right)$ & Momentum loss $(\xi)$ \\
\hline Low Mass & $\mathrm{p}+\mathrm{T} 2$ opposite only (no T1) & $3.4-8 \mathrm{GeV}$ & $2 \cdot 10^{-7}-10^{-6}$ \\
Medium Mass & $\mathrm{p}+\mathrm{T} 2$ opposite + T1 opposite & $8-350 \mathrm{GeV}$ & $10^{-6}-0.25 \%$ \\
High Mass & $\mathrm{p}+\mathrm{T} 2$ opposite + T1 same & $0.35-1.1 \mathrm{TeV}$ & $0.25 \%-2.5 \%$
\end{tabular}

arms). The single diffractive (SD) background was estimated completely from data using a SD dominated control sample (with charged particles in only one T2 arm and vetoing on charged particles anywhere in T1) with a single proton in the RPs. A significant background is due to internal "migration" i.e. real DD events that have their $\left|\eta^{+}\right|_{\min }$ and/or $\left|\eta^{-}\right|_{\min }$ in the uninstrumented region of $|\eta|<3.1$, where $\left|\eta^{+}\right|_{\min }\left(\left|\eta^{-}\right|_{\min }\right)$ refers to the primary particle with smallest $|\eta|$ in the positive (negative) hemisphere. This correction was estimated based on MC.

The forward double diffractive cross section was found to be $\sigma_{\mathrm{DD}}\left(4.7<\left|\eta^{ \pm}\right|_{\min }<6.5\right)=$ $116 \pm 25 \mu \mathrm{b}$. To increase the sensitivity to the differential $\left|\eta^{ \pm}\right|_{\min }$ distribution, the measurement was also repeated in combinations of smaller ranges of $\left|\eta^{ \pm}\right|_{\text {min }}$. All results are reported in table $\mathbb{W}$ and compared to prediction of MC models. Despite the limited $\left|\eta^{ \pm}\right|_{\min }$ coverage, the result indicates factorisation breaking in DD scattering at LHC i.e. $\sigma_{\mathrm{DD}}\left(4.7<\left|\eta^{ \pm}\right|_{\min }<6.5\right) \gg$ $\left.\sigma_{\mathrm{SD}}\left(4.7<\left|\eta^{+}\right|_{\min }<6.5\right) \cdot \sigma_{\mathrm{SD}}\left(-4.7>-\left|\eta^{-}\right|_{\min }>-6.5\right) / \sigma_{\mathrm{el}}\right)[\mathbb{W}]$. Using data taken in common with CMS at $\sqrt{s}=8 \mathrm{TeV}$ in 2012, a much larger fraction of $\sigma_{\mathrm{DD}}$ can be measured with good purity.

A study of SD events was performed on the same data set triggered with T2. SD-like (proton + gap + diffractive system) events were selected requiring only one proton in the RPs, charged particles in the T2 arm opposite to the proton and none in the other arm. The events were classified according to their diffractive mass, $M_{\text {diff }}$, based on the charged particle configuration in T1 and T2 (see table 2). Here the relation $M_{\text {diff }}^{2}=s \cdot e^{-\Delta \eta}$ was used, where $\Delta \eta$ is the rapidity gap between the very forward proton and the charged particle in $\mathrm{T} 1$ and $\mathrm{T} 2$ closest in $\eta$ to the proton.

The raw rate was first corrected for T2 trigger efficiency, RP acceptance and the shower probability of proton in the RP. Then the two-proton background from central diffraction and the pileup of a proton in RP from the beam halo (estimated from zero bias events) with a non-related inelastic event in T2 was subtracted, to obtain an estimate of the SD cross-section. The analysis was done separately for each set of near-far RPs (left arm top RP, left arm bottom RP, right arm top RP and right arm bottom RP) and the results were found to be consistent. The differential SD cross section with respect to $|t|, \mathrm{d} \sigma_{\mathrm{SD}} / \mathrm{d} t$, was also determined for the different classes. The distributions were fitted with an exponential, $A e^{-B|t|}$ and the $B$ parameter was found to decrease for larger diffractive masses. The aim of the study is to determine the $\sigma_{\mathrm{SD}}$ and $\mathrm{d} \sigma_{\mathrm{SD}} / \mathrm{d} t$ for each class separately.

\section{Forward multiplicities}

The T2 is an efficient tagger for an almost non-biased sample of inelastic events due to its low $p_{\mathrm{T}}$ acceptance threshold $(\sim 40 \mathrm{MeV} / \mathrm{c})$ and large acceptance to inelastic events $(>90 \%)$. Based on a special low-pileup run with $\beta^{*}=3.5 \mathrm{~m}$ at $\sqrt{s}=7 \mathrm{TeV}$ in 2011 , the charged particle pseudorapidity density $\mathrm{dN}_{\mathrm{ch}} / \mathrm{d} \eta$ for $5.3<|\eta|<6.4$ was determined in events with at least one charged particle 


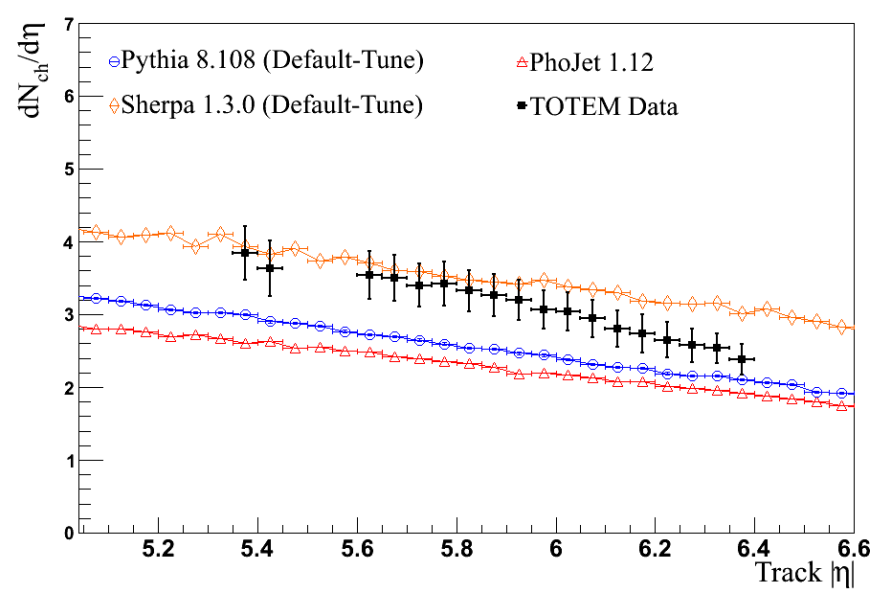

Figure 4: The $p_{\mathrm{T}}>40 \mathrm{MeV} / \mathrm{c}$ charged particle pseudorapidity density in events with at least one charged particle with $p_{\mathrm{T}}>40 \mathrm{MeV} / \mathrm{c}$ in the $5.3<|\eta|<6.5$ range compared the MC event generator predictions.

with transverse momentum above $40 \mathrm{MeV} / \mathrm{c}$ in this same pseudorapidity range [12]. The $\mathrm{dN}_{\mathrm{ch}} / \mathrm{d} \eta$ is found to decrease with $|\eta|$ as shown by Fig. 因. This was the first measurement of $\mathrm{dN}_{\mathrm{ch}} / \mathrm{d} \eta$ at such forward rapidities and provided useful input for the tuning for the $\mathrm{MC}$ event generators.

To further test the tuning as well as the underlying models of the MC event generators, TOTEM is pursuing together with CMS, a simultaneous measurement of the $\mathrm{dN}_{\mathrm{ch}} / \mathrm{d} \eta$ on the same events both for the central tracker $(|\eta|<2.3)$ and for the very forward T2 $(5.3<|\eta|<6.4)$. This measurement uses data taken in common in a low-pileup run at $\sqrt{s}=8 \mathrm{TeV}$ with $\mathrm{T} 2$ triggering both TOTEM and CMS. The measurement is pursued for three different samples to even further test the MC generators: an inclusive sample with at least a primary $\mathrm{T} 2$ track candidate and samples enhanced and depleted in single diffraction with at least one T2 primary track candidate only in one $\mathrm{T} 2$ arm and in both $\mathrm{T} 2$ arms, respectively. The final results are expected to be published soon.

\section{References}

[1] G. Anelli et al. (TOTEM Collaboration), JINST 3 (2008) S08007.

[2] S. Chatrchyan et al. (CMS Collaboration), JINST 3 (2008) S08004.

[3] G. Antchev et al. (TOTEM Collaboration), EPL 95 (2011) 41001.

[4] G. Antchev et al. (TOTEM Collaboration), EPL 96 (2011) 21001.

[5] J.R. Cudell et al. (COMPETE Collaboration), Phys. Rev. Lett. 89 (2002) 201801.

[6] G. Antchev et al. (TOTEM Collaboration), EPL 101 (2013) 21002.

[7] G. Antchev et al. (TOTEM Collaboration), EPL 101 (2013) 21003.

[8] G. Antchev et al. (TOTEM Collaboration), EPL 101 (2013) 21004.

[9] G. Antchev et al. (TOTEM Collaboration), Phys. Rev. Lett. 111 (2013) 012001.

[10] G. Antchev et al. (TOTEM Collaboration), CERN-PH-EP-2013-170 (2013), arXiv:1308.6722.

[11] V.A. Khoze, A.D. Martin and M.G. Ryskin, to be published.

[12] G. Antchev et al. (TOTEM Collaboration), EPL 98 (2012) 31002. 